\title{
TWO-WEIGHT INEQUALITIES FOR HARDY OPERATOR AND COMMUTATORS
}

\section{Wenming Li, Tingting Zhang And Limei Xue}

Abstract. For the maximal operator $N$ related to the Hardy operator $P$ and its adjoint $Q$, we give the characterizations for weights $(u, v)$ such that $N$ is bounded from $L^{p}(v)$ to $L^{p, \infty}(u)$ and from $L^{p}(v)$ to $L^{p}(u)$ respectively. We also obtain some $A_{p}$ type conditions which are sufficient for the two-weight inequalities for the Hardy operator $P$, the adjoint operator $Q$ and the commutators of these operators with CMO functions.

Mathematics subject classification (2010): 42B25, 40A30.

mal function.

Keywords and phrases: Hardy operator, Calderón operator, commutator, two-weight inequality, maxi-

\section{REFERENCES}

[1] J. Bastero, M. Milman and F. J. Ruiz, On the connection between weighted norm inequalities, commutators and real interpolation, Mem. Amer. Math. Soc., 154, (2001), no. 731.

[2] J. BRADley, Hardy inequalities with mixed norms, Canad. Math. Bull., 21, (4) (1978), 405-408.

[3] M. I. A. Cañestro, P. O. Salvador and C. R. Torreblanca, Weighted bilinear Hardy inequalities, J. Math. Anal. Appl., 387, (2012), 320-334.

[4] D. CRUZ-URIBE SFO, New proofs of Two-weight norm inequalities for the maximal operator, Georgian Math. J., 7, (2000), 33-42.

[5] J. García-Cuerva, And J. M. Martell, Two-weight norm inequalities for maximal operators and fractional integrals on non-homogeneous spaces, Indiana Univ. Math. J., 50, (2001), 1241-1280.

[6] J. García-Cuerva And J. L. Rubio de Francia, Weighted Norm Inequalities and Related opics, North-Holland Math. Stud., 116, 1985.

[7] J. Duonndikoetexa, F. J. Marttn-Reyes And S. Ombrosi, Calderón weights as Muckenhoupt weights, Indiana Univ. Math. J., 62, (2013), 891-910.

[8] G. H. HARDY, Note on a theorem of Hilbert, Math. Z., 6, (1920), 314-317.

[9] G. H. HARDY, Note on some points in the integral calculus, Messenger Math., 57, (1928), 12-16.

[10] G. H. Hardy, J. E. Littlewood and G. Polya, Inequalities, Cambridge University Press, Cambridge, UK, 1959.

[11] A. Kufner And L. E. Persson, Weighted Inequalities of Hardy Type, World Scientific, 2003.

[12] S. Long And J. WAng, Commutator of Hardy operators, J. Math. Anal. Appl., 274, (2002), 626644.

[13] V. G. MaZ’YA, Sobolev Spaces, Springer-Verlage, 1985.

[14] B. Muckenhoupt, Hardy's inequality with weights, Studia Math., 44, (1972), 31-38.

[15] B. OpIC, A. KufNER, Hardy-type Inequalities, Longman, 1990. 